\title{
BNIP3 is essential for mitochondrial bioenergetics during adipocyte remodelling in mice
}

\author{
Jin Woo Choi ${ }^{1,2}$ - Anna Jo ${ }^{1,3} \cdot$ Min Kim ${ }^{1}$ - Ho Seon Park ${ }^{1,4}$ - Sung Soo Chung ${ }^{1}$. \\ Shinae Kang ${ }^{4,5}$ • Kyong Soo Park ${ }^{1,3}$
}

Received: 8 October 2015 / Accepted: 13 November 2015 / Published online: 22 December 2015

(C) Springer-Verlag Berlin Heidelberg 2015

\begin{abstract}
Aims/hypothesis Adipose tissue is a highly versatile system in which mitochondria in adipocytes undergo significant changes during active tissue remodelling. BCL2/adenovirus E1B $19 \mathrm{kDa}$ protein-interacting protein 3 (BNIP3) is a mitochondrial protein and a known mitochondrial quality regulator. In this study, we investigated the role of BNIP3 in adipocytes, specifically under conditions of peroxisome proliferator-activated receptor- $\gamma(\operatorname{PPAR} \gamma)$-induced adipose tissue remodelling.

Methods The expression of BNIP3 was evaluated in 3T3-L1 adipocytes in vitro, C57BL/6 mice fed a high-fat diet and $d b / d b$ mice in vivo. Mitochondrial bioenergetics was investigated in BNIP3-knockdown adipocytes after rosiglitazone treatment. A putative peroxisome proliferator hormone responsive element (PPRE) was characterised by promoter assay and electrophoretic mobility shift assay (EMSA).
\end{abstract}

Jin Woo Choi and Anna Jo contributed equally to this study

Shinae Kang and Kyong Soo Park contributed equally as corresponding authors

Electronic supplementary material The online version of this article (doi:10.1007/s00125-015-3836-9) contains peer-reviewed but unedited supplementary material, which is available to authorised users.

Shinae Kang

shinae95@yuhs.ac

Kyong Soo Park

kspark@snu.ac.kr

1 Department of Internal Medicine, College of Medicine, Seoul National University, 101 Daehak-ro, Jongno-gu, Seoul 03080, South Korea

2 Department of Biomedical Sciences, College of Medicine, Seoul National University, Seoul, South Korea
Results The protein BNIP3 was more abundant in brown adipose tissue than white adipose tissue. Furthermore, BNIP3 expression was upregulated by 3T3-L1 pre-adipocyte differentiation, starvation and rosiglitazone treatment. Conversely, BNIP3 expression in adipocytes decreased under various conditions associated with insulin resistance. This downregulation of BNIP3 was restored by rosiglitazone treatment. Knockdown of BNIP3 in adipocytes inhibited rosiglitazoneinduced mitochondrial biogenesis and function, partially mediated by the 5' AMP-activated protein kinase (AMPK)-peroxisome proliferator-activated receptor $\gamma$, co-activator $1 \alpha$ $(\mathrm{PGC1} \alpha)$ signalling pathway. Rosiglitazone treatment increased the transcription level of Bnip 3 in the reporter assay and the presence of the PPRE site in the Bnip 3 promoter was demonstrated by EMSA.

Conclusions/interpretation The protein BNIP3 contributes to the improvement of mitochondrial bioenergetics that occurs

3 Department of Molecular Medicine and Biopharmaceutical Sciences, Graduate School of Convergence Science and Technology, Seoul National University, Seoul, South Korea

4 Department of Internal Medicine, Gangnam Severance Hospital, Yonsei University College of Medicine, 211 Eonju-ro (146-92 Dogok-dong), Gangnam-gu, Seoul 135-710, South Korea

5 Severance Institute for Vascular and Metabolic Research, Yonsei University College of Medicine, Seoul, South Korea 
on exposure to rosiglitazone. It may be a novel therapeutic target for restoring mitochondrial dysfunction under insulinresistant conditions.

Keywords Adipocytes · Bioenergetics · BNIP3 - Insulin resistance $\cdot$ Mitochondria $\cdot \mathrm{PPRE} \cdot$ Rosiglitazone

\begin{tabular}{|c|c|}
\hline \multicolumn{2}{|c|}{ Abbreviations } \\
\hline BAT & Brown adipose tissue \\
\hline BNIP3 & $\begin{array}{l}\text { BCL2/adenovirus E1B } 19 \mathrm{kDa} \text { protein- } \\
\text { interacting protein } 3\end{array}$ \\
\hline BODIPY & Boron-dipyrromethene \\
\hline $\mathrm{DIO}$ & Diet-induced obesity \\
\hline EMSA & Electrophoretic mobility shift assay \\
\hline LC3B & $\begin{array}{l}\text { Isoform B of microtubule-associated protein } 1 \\
\text { light chain } 3\end{array}$ \\
\hline mtDNA & Mitochondrial DNA \\
\hline OCR & Oxygen consumption rate \\
\hline $\operatorname{PPAR} \gamma$ & Peroxisome proliferator-activated receptor- $\gamma$ \\
\hline PPRE & Peroxisome proliferator responsive element \\
\hline RT-qPCR & Quantitative RT-PCR \\
\hline siRNA & Small interfering RNA \\
\hline siNS & Non-specific scramble siRNA \\
\hline SVF & Stromal vascular fraction \\
\hline TEM & Transmission electron microscopy \\
\hline WAT & White adipose tissue \\
\hline
\end{tabular}

\section{Introduction}

Adipocytes are one of the cell types that remodel dynamically in number and size [1]. Within adipocytes, mitochondria show robust changes in density and morphology under pathophysiological conditions [2, 3]. Excess mitochondria occur with myopathy or cancer [4] and reduced mitochondria number and/or function is associated with obesity [5-7]. This suggests that tight quality control of the mitochondria may be critical for maintaining adipocyte function during adipocyte remodelling.

Peroxisome proliferator-activated receptor- $\gamma(\operatorname{PPAR} \gamma)$ is a key transcription factor essential for adipocyte differentiation [8]. Agonists of PPAR $\gamma$ improve insulin resistance, probably by stimulating the storage of excess energy as fat, thus improving blood glucose [9]. Many studies have also reported that PPAR $\gamma$ agonists induce mitochondrial biogenesis $[2,7$, $10,11]$. Based on the critical role of mitochondria in energy generation, mitochondrial biogenesis may contribute to $\operatorname{PPAR} \gamma$-agonist-induced improvement in insulin resistance [12]. However, the molecular mechanisms of impaired mitochondrial function and restoration of mitochondrial bioenergetics by PPAR $\gamma$ agonists in obesity are still poorly understood.

Located at the outer membrane of mitochondria, BCL2/adenovirus E1B $19 \mathrm{kDa}$ protein-interacting protein 3 (BNIP3) was originally known as an inducer of apoptosis [13]. Additionally, BNIP3 is also an inducer of autophagyrelated cell survival [14]. Under certain stress conditions, such as hypoxia, BNIP3 controls mitochondrial quality by removing damaged mitochondria via autophagy/mitophagy, and protects cells from death [15]. This bidirectional action of BNIP3 between apoptosis and autophagy suggests that BNIP3 may be a key regulator of cell fate between cell death and survival during dynamic remodelling $[16,17]$.

The biological significance of BNIP3 has been investigated largely in myocytes and cancer cells [18, 19], cell types in which mitochondria are abundant. In terms of other metabolism-related effects in tissues, a group has reported that BNIP3-null mice livers have higher mitochondrial mass but impaired function compared with control mice [20]. Another study has shown that Bnip 3 transcription is upregulated during brown fat adipogenesis [21]. However, the role of BNIP3 in white adipose tissue (WAT), a mitochondria-poor tissue, has not been evaluated. Considering that a PPAR $\gamma$ agonist induces robust mitochondria biogenesis in adipocytes, we hypothesised that BNIP3 influences adipose tissue remodelling by regulating mitochondrial bioenergetics under rosiglitazone-treated conditions.

\section{Methods}

Animal experiments We used C57BLKS/J diabetic Lepr $(d b / d b)$ and lean control $(d b / m)$ mice (SLC, Shizuoka, Japan) and C57BL/6 mice (Orient Bio, Seongnam, Gyeonggi-do, Korea) for in vivo experiments. The control group was fed standard rodent chow (PMI LabDiet; Purina Mills, St Louis, MO, USA) and the rosiglitazone-treated group was fed a mixture of standard chow and rosiglitazone maleate (GlaxoSmithKline, Brentford, UK). From 9 weeks of age, $30 \mathrm{mg} \mathrm{kg}{ }^{-1}$ day $^{-1}$ rosiglitazone was included in chow for 4 weeks. For the diet-induced obesity (DIO) model, C57BL/ 6 mice were fed $60 \%$ high-fat chow (PMI LabDiet) for 8 weeks. For the starvation model, C57BL/6 mice were starved for $48 \mathrm{~h}$. Male mice, 8 to 12 weeks of age, were used for experiments. All animals were randomised according to body weight and all experiments carried out with animal samples were blinded to group assignment. All animal studies were approved by the Institutional Animal Care and Use Committee of Seoul National University Hospital. For isolation of adipocyte fraction and stromal vascular fraction (SVF) methods, see the electronic supplementary material (ESM) Methods. 
Mitochondrial and cytosolic fractionation of cells The mitochondrial fraction was isolated by gradient difference. See the ESM Methods for further details.

Cell culture and treatment Cultured 3T3-L1 pre-adipocytes (gift of J.-W. Kim, Yonsei University College of Medicine, Seoul, Korea) were maintained in DMEM and differentiated by treatment with dexamethasone, 3-isobutyl-1methylxanthine and insulin. For some experiments, we added $1 \mu \mathrm{mol} / 1$ rosiglitazone or $50 \mathrm{ng} / \mathrm{mL} \mathrm{TNF}-\alpha$ (R\&D systems, Minneapolis, MN, USA) to the differentiated 3T3-L1 cells for $72 \mathrm{~h}$. For hypoxic cell culture, we cultivated cells in an incubator filled with $1 \% \mathrm{O}_{2}$ for $24 \mathrm{~h}$. Cells were confirmed to be negative for mycoplasma. For further details, see the ESM Methods.

Western blot analysis Western blots were analysed using the antibodies indicated. See the ESM Methods for further details.

RNA/DNA preparation and PCR Isolation of total RNA and semi-quantitative or quantitative RT-PCR (RT-qPCR) were performed according to the guideline [22] with appropriate primers and Taq DNA polymerase (Qiagen, Valencia, CA, USA) or SYBR Premix Ex Taq reagents (Takara Bio, Shiga, Japan), respectively. The same process was followed for mitochondrial (mt)DNA copy number evaluation. See the ESM Methods for further details.

Imaging analysis We evaluated the adipocyte morphology using a confocal microscope after whole-mount immunostaining and also by transmission electron microscopy (TEM). See the ESM Methods for further details.

Fluorescence-activated cell sorter analysis To assess mitochondrial mass, we performed fluorescence-activated cell sorter (FACS) analysis. Mitochondrial mass was measured by fluorescence levels on staining with MitoTracker Green FM (Molecular Probes, Life Technologies, Carlsbad, CA, USA). A FACSCalibur flow cytometer (BD, Franklin Lakes, NJ, USA) was used for quantitative FACS analysis. See the ESM Methods for further details.

Oxygen consumption rate To determine cellular oxygen consumption in fully differentiated 3T3-L1 adipocytes, the Seahorse Extracellular Flux Analyzer XF24 (Seahorse Bioscience, Billerica, MA, USA) was used [23, 24].

Bnip3 promoter reporter assays The $-2,874$ putative peroxisome proliferator hormone responsive element (PPRE) and mutated form of PPRE were introduced into the pGL2P vector in triple repeated form (pGL2P-Bnip3-PPREwt and pGL2PBnip3-PPREmt, respectively). Transient transfection of Cos7 cells with $0.3 \mu \mathrm{g}$ reporter vector (pGL2P-Bnip3-PPREwt or
pGL2P-Bnip3-PPREmt), $0.1 \mu \mathrm{g}$ expression vectors for PPAR $\gamma$ and $0.05 \mu \mathrm{g}$ pCMV- $\beta$-galactosidase vector was carried out using Lipofectamine Plus reagent (Invitrogen). We treated the cells with rosiglitazone $(1 \mu \mathrm{mol} / \mathrm{l})$ for $24 \mathrm{~h}$ and measured luciferase activity. See the ESM Methods for further details.

Electrophoretic mobility shift assay We constructed oligonucleotides for PPRE from $-2,874$ to $-2,861$ in the Bnip3 promoter region for electrophoretic mobility shift assay (EMSA) probes and then performed binding and competition assays to determine the electrophoretic mobility shift. See the ESM Methods for further details.

Statistical analysis Statistical analysis of the data was done with SPSS version 17.0 (SPSS, Chicago, IL, USA). An independent Student's $t$ test was used to measure the differences between means. Data have been expressed as mean \pm SE. A $p$ value $<0.05$ was considered statistically significant. None of the data were excluded on the basis that the results do not support the main findings.

\section{Results}

BNIP3 expression is actively regulated in relation to change of mitochondrial bioenergetics As BNIP3 is known to induce apoptosis/autophagy through mitochondrion-related signalling, we evaluated the expression pattern of BNIP3 under conditions of active mitochondrial turnover. The gene Bnip3 was upregulated from day 2 after differentiation induction and peaked at day 4 in 3T3-L1 adipocytes (Fig. 1a-d). The BNIP3 expression pattern closely followed the PPAR $\gamma$ expression pattern (Fig. 1c). The level of BNIP3 was markedly upregulated in the adipose tissue after starvation (Fig. 1e, f), in brown adipose tissue (BAT; interscapular fat) (Fig. 1g, h) and by rosiglitazone treatment (Fig. 1i, j) in vivo. More specifically, Bnip3 upregulation by rosiglitazone occurred mainly in the adipocyte fraction and not in the SVF (containing preadipocytes, macrophages and endothelial cells) (Fig. 1k). We also evaluated the pattern of BNIP3 expression in adipose tissue from mouse models of obesity. The protein level of BNIP3 was reduced in DIO (Fig. 11, m) and $d b / d b$ mice (Fig. 1n, o) compared with control mice. Reduced BNIP3 in the adipose tissue of $d b / d b$ mice occurred in adipocytes, but not in macrophages in the SVF (Fig. 1p). Additionally, BNIP3 was strongly downregulated in TNF- $\alpha$-treated adipocytes compared with vehicle-treated cells in vitro (Fig. 1q, r). Overall, these findings show a considerable amount of BNIP3 is present in adipocytes and actively regulated by various stimuli related to mitochondrial bioenergetics. 

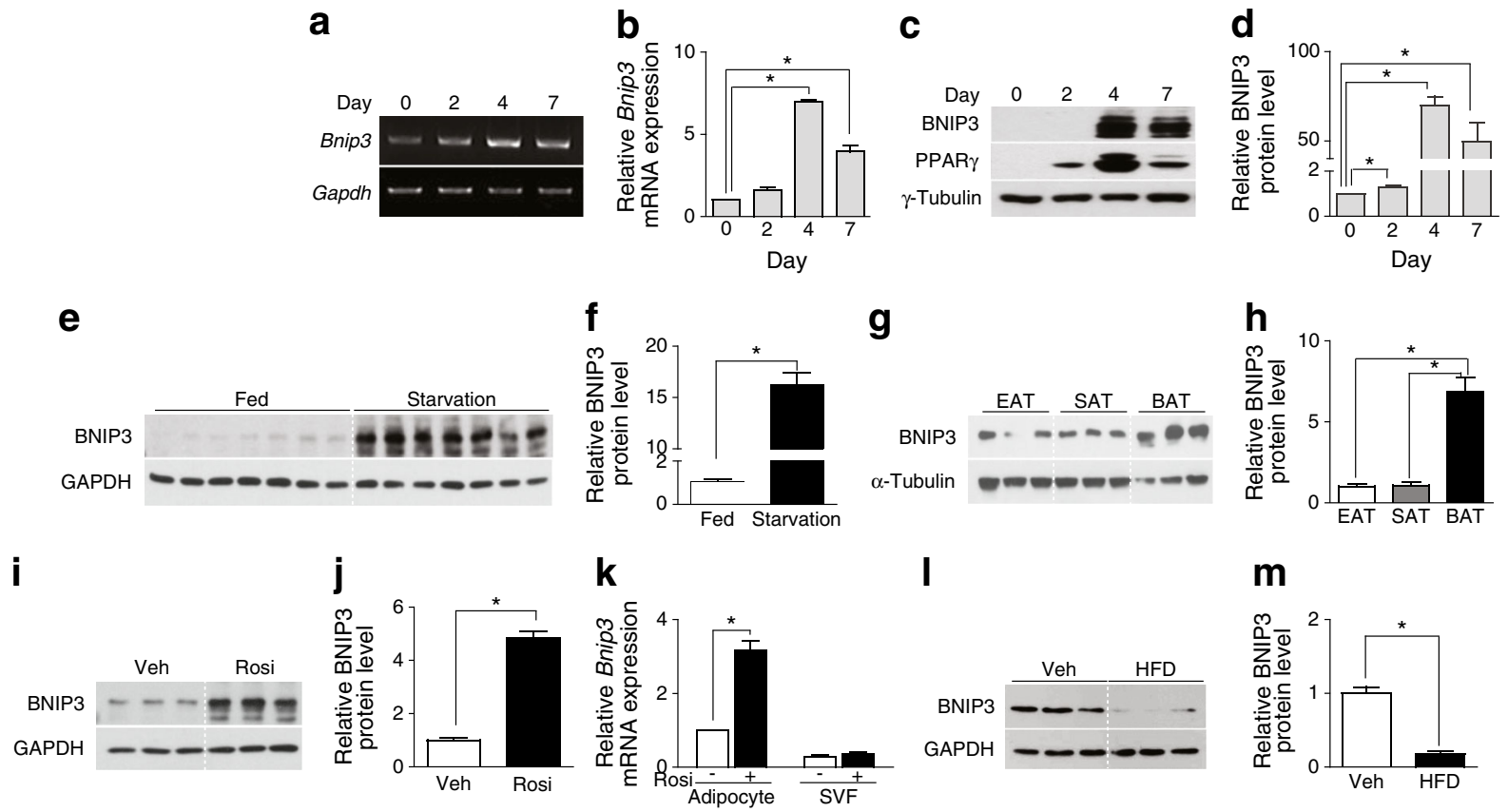

I
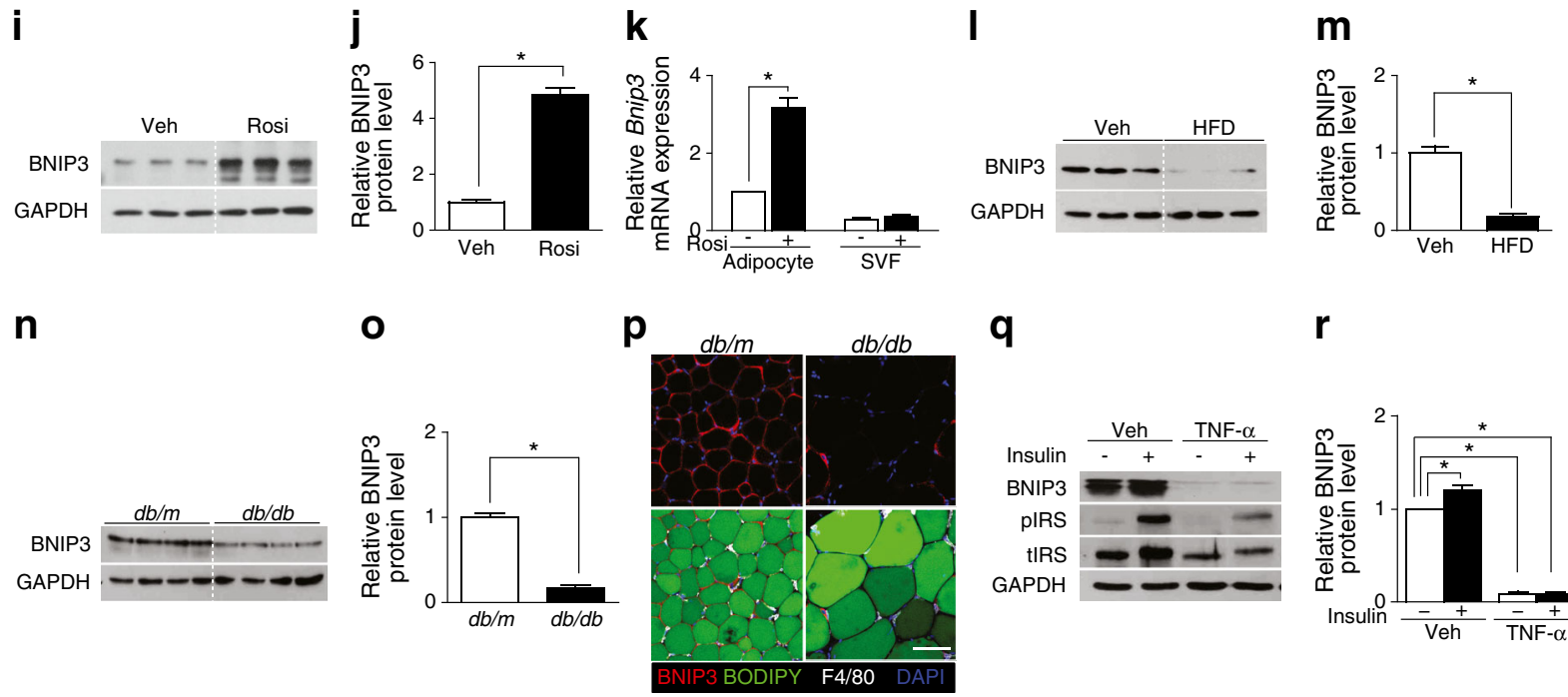

q

$\mathbf{r}$
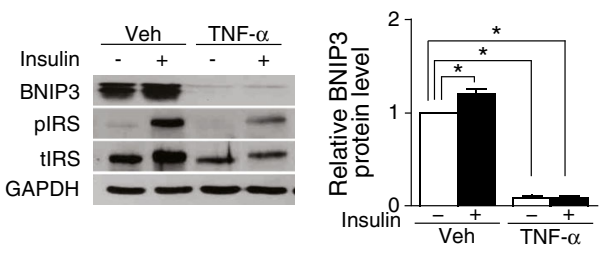

Fig. 1 BNIP3 expression is actively regulated during adipocyte remodelling and downregulated in the obese diabetic condition. (a-d) 3T3-L1 pre-adipocytes were harvested during induction of differentiation at the times indicated. (a) Levels of mRNA from semi-quantitative PCR and (b) RT-qPCR, and (c, d) protein levels of BNIP3 and PPAR $\gamma(n=4) .(\mathbf{e}, \mathbf{f})$ Epididymal fat after $48 \mathrm{~h}$ starvation $(n=7)$ and $(\mathbf{g}, \mathbf{h})$ white and brown fat samples $(n=6)$ were harvested from C57BL/6 mice. (i, j) Epididymal adipose tissue was harvested after 1 week of exposure to rosiglitazone or vehicle $(n=10)$. (k) Epididymal adipose tissue was separated into adipocyte and SVF fractions and Bnip3 mRNA was analysed with RT-qPCR $(n=4)$. (1-p) Epididymal adipose tissue was harvested from mice fed a high-fat diet or vehicle (1, m; $n=9)$ and from $\mathrm{db} / \mathrm{db}$ or $\mathrm{db} / \mathrm{m}$ mice (n, $\mathbf{0} ; n=11)$ and (1-o) BNIP3 levels were evaluated on immunoblots. (p) Whole adipose tissue immunostaining with antibodies to BNIP3 and F4/80, and boron-dipyrromethene (BODIPY) and DAPI. Scale bar, $100 \mu \mathrm{m}$. (q, r) Differentiated 3T3-L1 adipocytes were treated with TNF$\alpha$ or vehicle $(n=5)$. Density quantification $(\mathbf{d}, \mathbf{f}, \mathbf{h}, \mathbf{j}, \mathbf{m}, \mathbf{o}, \mathbf{r})$ and $(\mathbf{b}, \mathbf{k})$ mRNA expression of Bnip 3 are presented as relative values after normalisation to each loading or reference gene and compared with the reference value set at 1 . Data are presented as mean \pm SE. ${ }^{*} p<0.05$. EAT, epididymal adipose tissue; GAPDH, glyceraldehyde-3-phosphate dehydrogenase; HFD, high-fat diet; rosi, rosiglitazone; veh, vehicle
BNIP3 downregulation is restored by a PPAR $\gamma$ agonist in adipocytes in the obese diabetic condition We examined whether rosiglitazone treatment could restore the BNIP3 decrement in $d b / d b$ mice. After 4 weeks of rosiglitazone treatment, the BNIP3 level in $d b / d b$ mice increased comparably with that of the rosiglitazone-treated $\mathrm{db} / \mathrm{m}$ mice (Fig. 2a, b). Restoration of BNIP3 expression by rosiglitazone treatment in $\mathrm{db} / \mathrm{db}$ mice was primarily confined to the cytoplasm of adipocytes (Fig. 2c). In addition, BNIP3 levels were lower in whole adipose tissue and in the adipocyte fraction in tissue from $d b / d b$ mice compared with $d b / m$ mice, with levels increased by rosiglitazone treatment (Fig. 2d, e). Rosiglitazone treatment also restored the TNF- $\alpha$-induced BNIP3 decrease in 3T3-L1 adipocytes (Fig. 2f, g). Next, we evaluated the amount of BNIP3 in the mitochondrial fraction after dividing the lysates from whole adipose tissue in mice and from 3T3-L1 adipocytes into cytosolic and mitochondrial proteins. The expression of BNIP3 per equal amount of mitochondrial protein increased with rosiglitazone treatment both in vivo (Fig. 2h) and in vitro (Fig. 2i).

BNIP3 is a critical molecule for enhancing rosiglitazoneinduced mitochondrial bioenergetics Next, we investigated the role of BNIP3 in 3T3-L1 adipocytes, especially in 
Fig. 2 BNIP3 downregulation is restored by a PPAR $\gamma$ agonist in adipocytes in the obese diabetic condition. (a-e, h) Epididymal adipose tissue was harvested after rosiglitazone or vehicle administration in $d b / d b$ or $d b / m$ mice (a, b: $n=6-8 ; \mathbf{d}, \mathbf{e}: n=3-4)$. (f, g, i) Differentiated 3T3-L1 adipocytes treated with TNF- $\alpha$ or vehicle with or without insulin (f, g), or transfected with siBnip3 or siNS (i) were treated with or without rosiglitazone $(n=4)$. (h) Whole adipose tissue $(n=4)$ or $(\mathbf{i})$ 3T3-L1 adipocytes $(n=5)$ were fractionated into cytosolic and mitochondrial components. (a, f, h, i) BNIP3 immunoblotting. (c) BNIP3 and BODIPY

immunostaining $(n=4)$. Scale bar, $100 \mu \mathrm{m}$. (d, e) Bnip3 mRNA analysis with RT-qPCR. Density quantification $(\mathbf{b}, \mathbf{g})$ and mRNA expression $(\mathbf{d}, \mathbf{e})$ of BNIP3 are presented as relative values after normalisation to each loading or reference gene and compared with the reference value set at 1 . Data presented as mean $\pm \mathrm{SE}$. ${ }^{*} p<0.05$. Cyto, cytosolic fraction; GAPDH, glyceraldehyde-3phosphate dehydrogenase; Mito, mitochondrial fraction; Rosi, rosiglitazone; veh, Vehicle a
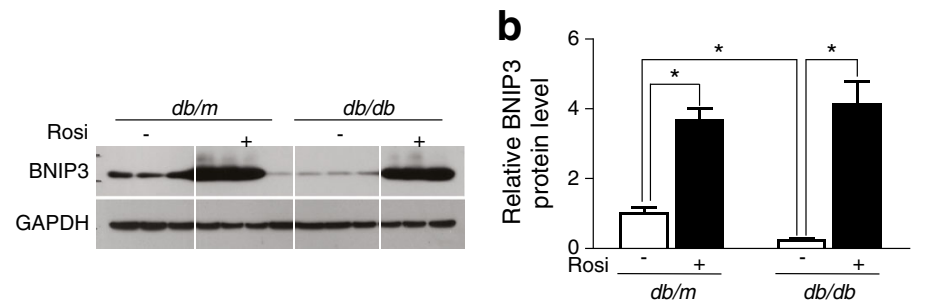

C

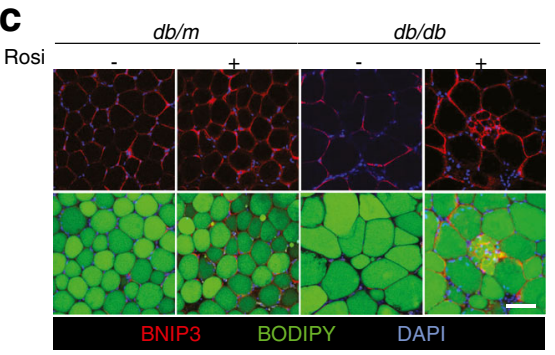

d

e
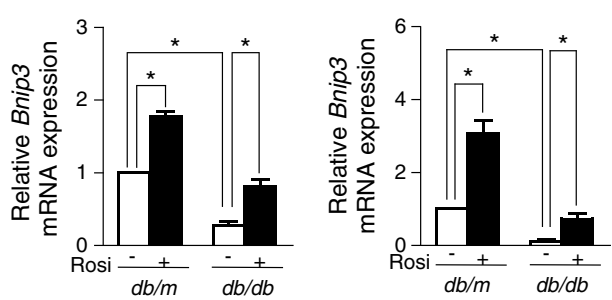

f
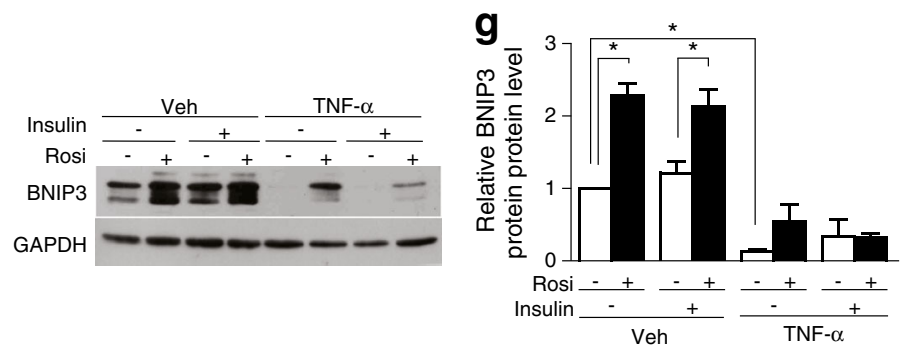

h

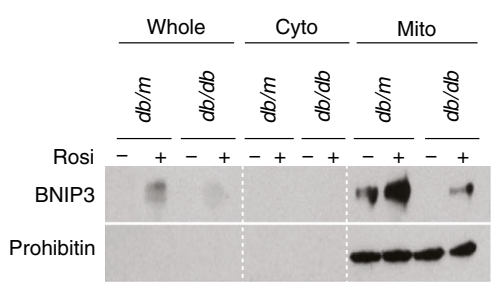

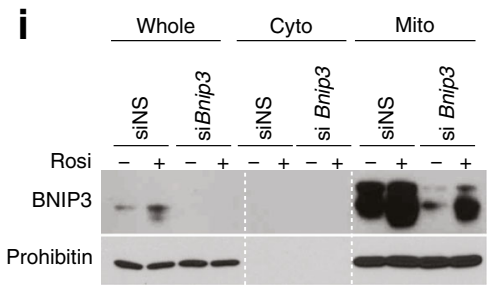

rosiglitazone-induced adipocyte remodelling in vitro. Using FACS analysis after tagging mitochondria with MitoTracker, we observed that rosiglitazone treatment increased mitochondrial mass in control adipocytes, in contrast to little increase in adipocytes treated with rosiglitazone after BNIP3 knockdown (Fig. 3a). Additionally, rosiglitazone increased relative mtDNA copy number and Tfam and Nrf1 mRNA levels, all of which were attenuated in BNIP3-knockdown adipocytes exposed to rosiglitazone (Fig. 3b-d). However, there were no significant differences in the above variables between the BNIP3-knockdown and the control adipocytes without rosiglitazone treatment. Rosiglitazone also increased ATP content in non-specific scramble small interfering (si)RNA ( iNS)-transfected adipocytes, whereas it could not in siBnip3-transfected adipocytes (Fig. 3e). In TEM images, the number of small dense mitochondria was increased by rosiglitazone treatment in siNS-transfected adipocytes. However, siBnip3-transfected adipocytes showed a heterogeneous mix of small and elongated mitochondria after rosiglitazone treatment (Fig. 3f). Additionally, BNIP3 knockdown significantly attenuated the rosiglitazone-induced upregulation of the maximal respiration rate. Also, BNIP3 knockdown tended to reduce the increase in energy expenditure (the difference in oxygen consumption rate [OCR] with oligomycin and rotenone) stimulated by rosiglitazone exposure (Fig. $3 g$, h). The increase in the mRNA level of $U c p 1$ on rosiglitazone exposure that was seen in control cells was not apparent in cells with BNIP3 knockdown (Fig. 3i). This indicates that BNIP3 could be a critical molecule for controlling mitochondrial bioenergetics during rosiglitazone-induced mitochondrial remodelling.

BNIP3 contributes to mitochondrial bioenergetics by activation of the 5' AMP-activated protein kinase-peroxisome proliferator-activated receptor $\gamma$ co-activator $1 \alpha$ pathway, but not by autophagy induction We evaluated whether BNIP3 contributes to mitochondrial bioenergetics in PPAR $\gamma$ agonist-treated 3T3-L1 adipocytes via autophagy activation 
a

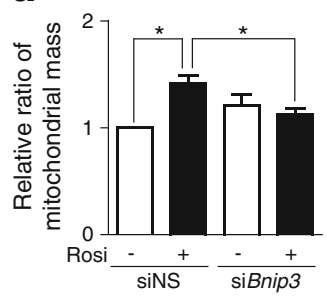

e

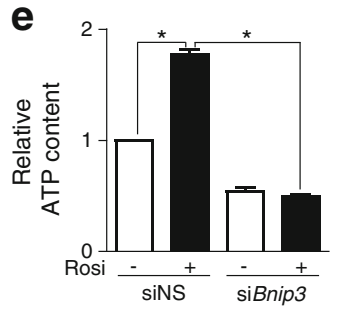

b

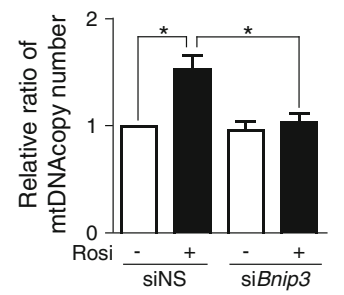

C

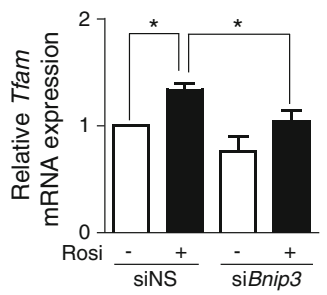

d

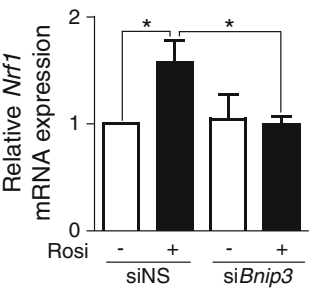

f

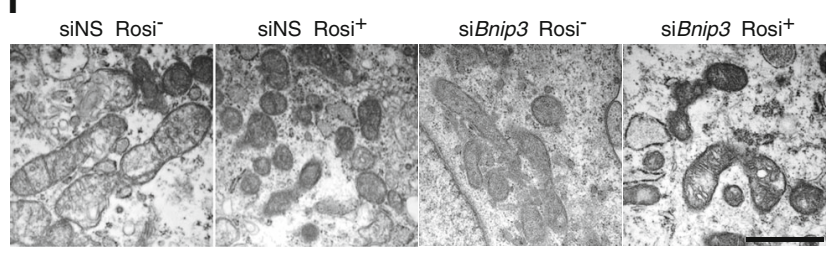

g

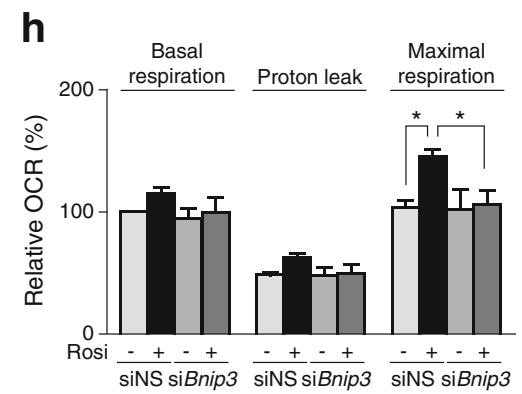

i
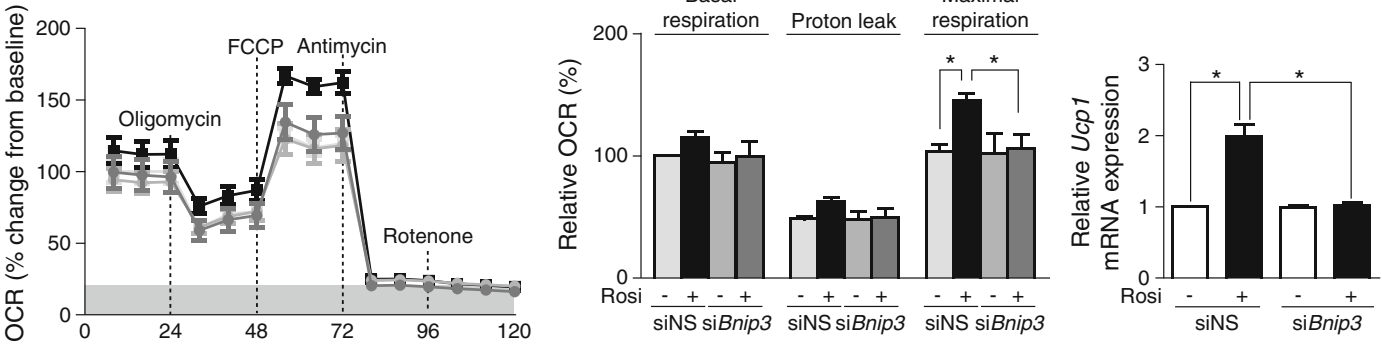

Fig. 3 BNIP3 is a critical molecule for enhancing rosiglitazone-induced mitochondrial bioenergetics. (a-i) 3T3-L1 adipocytes were transfected with siNS or siBnip3 and then treated with or without rosiglitazone. (a) FACS analysis for mitochondria with MitoTracker Green $(n=9)$. (b) Relative mtDNA copy number, (c) Tfam and (d) $N r f 1$ were evaluated by qPCR (b) or RT-qPCR (c, d) and normalised to $\beta$-actin from nuclearspecific DNA (b) or 18S RNA (c, d) $(n=5)$. (e) ATP content is presented as relative fluorescein count $(n=5)$. (f) Mitochondria were visualised by $\operatorname{TEM}(n=5)$. Scale bar, $1 \mu \mathrm{m}$. $(\mathbf{g}, \mathbf{h})$ OCR was measured using Seahorse after treatment with oligomycin, carbonyl cyanide-4(trifluoromethoxy)phenylhydrazone (FCCP), antimycin or rotenone and is presented as the relative value of basal respiration rate of siNS without rosiglitazone, set as $100 \%(n=5)$ (black rectangle, siNS treated; circle, siBnip treated; light grey, without rosiglitazone, dark grey with rosiglitazone). (h) Basal respiration, proton leak and maximal respiration were compared between groups. (i) Ucpl mRNA level was evaluated with RT-qPCR $(n=5)$. Data are presented as mean \pm SE compared with the reference value set at $1 . * p<0.05$. Rosi, rosiglitazone in vitro. Contrary to expectations, rosiglitazone treatment decreased autophagy, as shown by a decrease in LC3BII and an increase in p62 expression compared with vehicle-treated adipocytes. In TNF- $\alpha$-treated adipocytes where basal autophagy activity was elevated, rosiglitazone treatment also decreased autophagy (Fig. 4a, left panel). Additionally, hypoxia upregulated BNIP3 expression to a level comparable with that achieved with rosiglitazone treatment. Rosiglitazone treatment decreased autophagy even after BNIP3 upregulation under hypoxia, a phenomenon similar in cells treated with TNF- $\alpha$ under hypoxia (Fig. 4a, right panel). The 3T3-L1 adipocytes showed no significant change in LC3B after siBnip3 transfection, and LC3BII was decreased by rosiglitazone treatment independent of BNIP3 knockdown (Fig. 4b). These findings suggest that upregulation of BNIP3 by rosiglitazone might not induce autophagy, at least not in 3T3-L1 adipocytes.

To clarify how BNIP3 contributes to rosiglitazoneinduced mitochondrial biogenesis, we evaluated the expression of Pgcla (also known as Ppargcla), which encodes peroxisome proliferator-activated receptor $\gamma$ coactivator $1 \alpha$ and is the master gene regulating mitochondrial biogenesis. Production of $\mathrm{PGC} 1 \alpha$ was upregulated by rosiglitazone treatment, but this effect was attenuated by BNIP3 knockdown, suggesting that BNIP3 is critical in rosiglitazone-induced PGC1 $\alpha$ upregulation (Fig. 4c-e). We also investigated whether rosiglitazone-induced activation of $5^{\prime}$ AMP-activated protein kinase (AMPK), the upstream regulator of PGC $1 \alpha$, depends on BNIP3. Phosphorylation of AMPK by rosiglitazone was attenuated in siBnip3-transfected adipocytes, concordant with the attenuation of adiponectin expression by BNIP3 knockdown even after rosiglitazone stimulation (Fig. $4 \mathrm{f}-\mathrm{h}$ ). These data suggest that BNIP3 mediates rosiglitazone-induced mitochondrial biogenesis, probably through the AMPK-PGC $1 \alpha$ pathway. 
Fig. 4 BNIP3 partially contributes to mitochondrial bioenergetics by AMPK-PGC1 $\alpha$ pathway activation, but not by autophagy induction. (a)

Differentiated 3T3-L1 adipocytes were cultured under normoxia or hypoxia and treated as indicated $(n=4)$. (b-h) 3T3-L1 adipocytes were transfected with siNS or siBnip 3 and then treated with or without rosiglitazone. (a, b) BNIP3, LC3B and p62 immunoblotting $(n=4-5)$. Immunoblots for (c, d) PGC1 $\alpha$ $(n=4)$ and (f, g) pAMPK and tAMPK $(n=5)$. Analysis of mRNA for Pgcl $\alpha(n=6)(\mathbf{e})$ and adiponectin $(n=8)$ (h) by RT-qPCR. Density quantification $(\mathbf{d}, \mathbf{g})$ and mRNA $(\mathbf{e}, \mathbf{h})$ results are presented as relative values after normalisation to each loading or reference gene. Data are presented as mean $\pm \mathrm{SE}$ compared with the reference value set at 1 . ${ }^{*} p<0.05$. Rosi, rosiglitazone

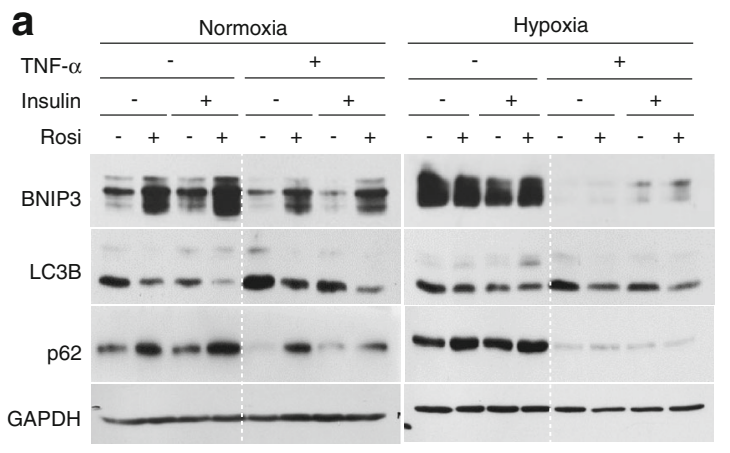

b

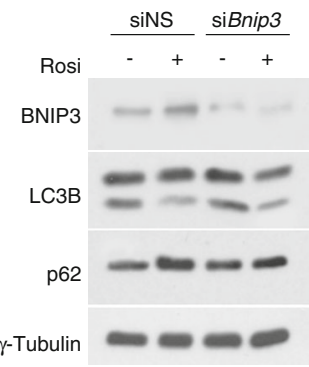

C

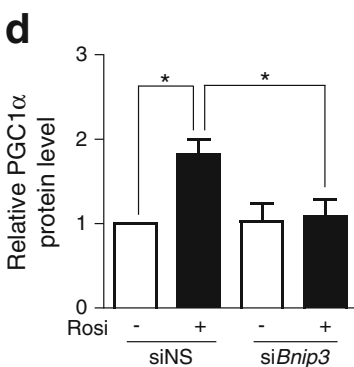

e

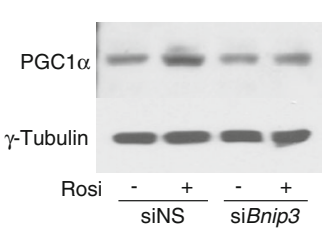

f

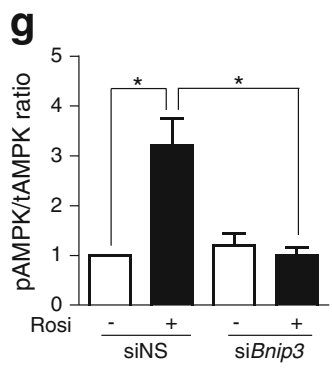

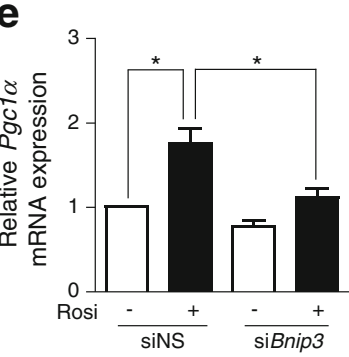

h

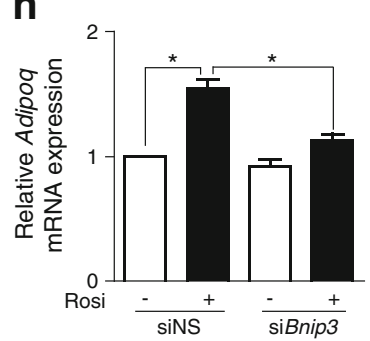

\section{Direct binding of PPAR $\gamma$ agonist to PPRE in its promoter} upregulates Bnip3 transcription Next, we evaluated the mechanism of BNIP3 upregulation by rosiglitazone in vitro. There were minimal changes in Hifl- $\alpha$ (also known as Hifla) transcription during rosiglitazone-induced BNIP3 upregulation (Fig. 5a, b). Transcription of Bnip3 significantly increased by twofold after $24 \mathrm{~h}$ of rosiglitazone treatment in differentiated 3T3-L1 adipocytes (Fig. 5c, d). Upregulation of Bnip3 by rosiglitazone diminished in adipocytes transfected with siPpar (also known as Pparg) (Fig. 5e), indicating that Bnip3 mRNA expression depends on PPAR $\gamma$ activity. When the PPRE activity was tested in the site from $-2,874$ to $-2,861$ bp in the Bnip3 promoter, which exhibited the greatest homology to the consensus PPRE sequence (Fig. 5f), the $-2,874$ site showed an approximately twofold increase in reporter luciferase activity with rosiglitazone treatment, whereas the mutant sequence of this site did not (Fig. $5 \mathrm{~g}, \mathrm{~h}$ ). In the binding affinity assay, the probe from $-2,874$ to $-2,861 \mathrm{bp}$ in the Bnip3 promoter bound to the same site as the consensus PPRE probe (Fig. 5i). In the competition assay, binding of the putative PPRE to the radiolabelled probe competed with the unlabelled self-competitors and the control consensus PPRE, but not with the mutant competitors, in a dose-dependent manner. Supershift resulting from the addition of PPAR $\gamma$-specific antibody further proved that the $-2,874$ to $-2,861$ bp region of the Bnip3 promoter is a PPAR $\gamma$ binding site (Fig. 5j). Together, these results indicate that BNIP3 is transcriptionally upregulated by the PPRE site in the Bnip3 promoter.

\section{Discussion}

In this study, we demonstrated that BNIP3 is a critical molecule for controlling mitochondrial bioenergetics in adipocytes. The level of BNIP3 was downregulated in adipocytes under diabetic obese conditions, but was restored by rosiglitazone treatment. Under adipocyte remodelling conditions, BNIP3 determined the fate of mitochondrial bioenergetics, probably by AMPK-PGC1 $\alpha$ signalling. Signalling via BNIP3 was directly mediated by the PPRE in the Bnip3 promoter. These findings suggest a critical new role for BNIP3 in the regulation of adipocyte remodelling, especially in the control of mitochondria. 
a

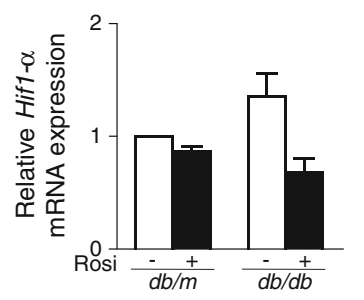

b

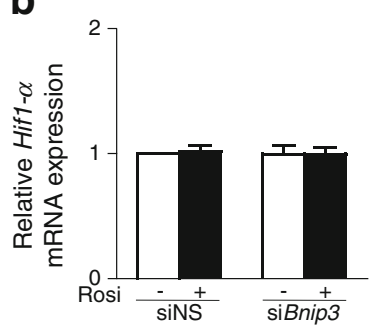

C

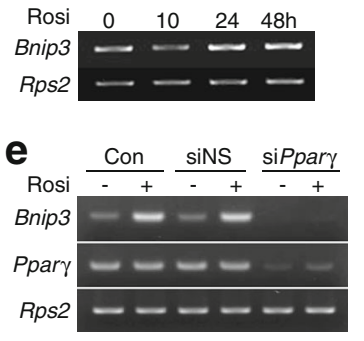

d

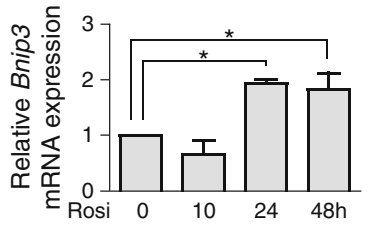

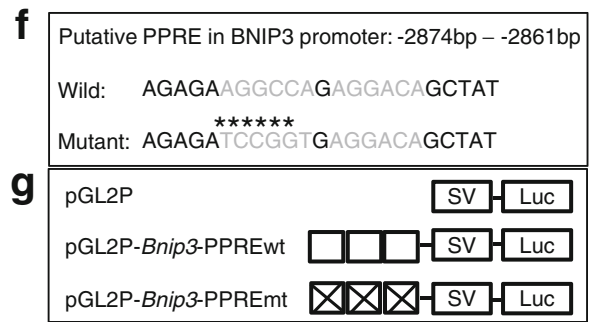

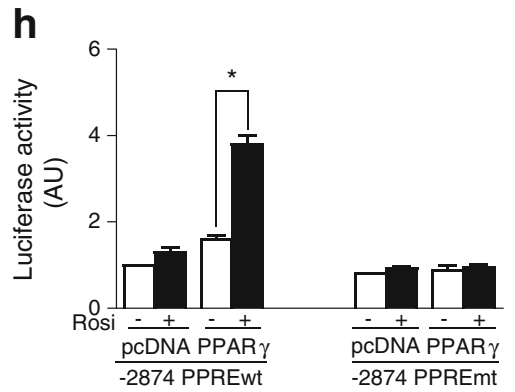

Fig. 5 Rosiglitazone transcriptionally upregulates BNIP3 through the PPRE in the Bnip3 promoter during adipocyte remodelling. (a) $d b / d b$ and $\mathrm{db} / \mathrm{m}$ mice were fed with rosiglitazone or vehicle and epididymal adipose tissue was harvested $(n=3)$. (b) Differentiated 3T3-L1 adipocytes were transfected with siNS or siBnip3 and then treated with or without rosiglitazone $(n=3)$. (a, b) Hif-1 $\alpha$ RT-qPCR. (c, d) 3T3-L1 adipocytes were treated with rosiglitazone over time. (c) Bnip3 semi-quantitative RTPCR $(n=4)$. (d) Bnip3 RT-qPCR $(n=3)$. (e) 3T3-L1 adipocytes were transfected with the indicated siRNA and each group was treated with rosiglitazone or vehicle. Semi-quantitative RT-PCR of Bnip3 and Ppar $(n=4)$. (f) Schematic diagram of the putative PPRE site. (g) The $-2,874$ PPRE or its mutated form was introduced into a pGL2P vector. (h) Luciferase activity was measured in COS-7 cells transfected with

\section{Regulation of BNIP3 during adipocyte remodelling, espe-} cially in obesity or PPAR $\gamma$-related conditions The role of BNIP3 has been mostly investigated in myocytes in relation to hypoxia-induced apoptotic cell death and autophagic cell survival [14]. Here, we revealed that a considerable amount of BNIP3 expression is regulated in adipocytes under conditions in which higher mitochondrial content is required, including adipocyte differentiation [10], starvation [25] and in BAT [26], and also by rosiglitazone treatment $[2,7,10]$. We also found that BNIP3 was downregulated in adipose tissue under obese diabetic conditions. Our findings that adipocytes with BNIP3 knockdown had attenuated mitochondrial biogenesis and ATP production, even after rosiglitazone treatment (Fig. 3), suggest that the BNIP3 decrement in obesity might

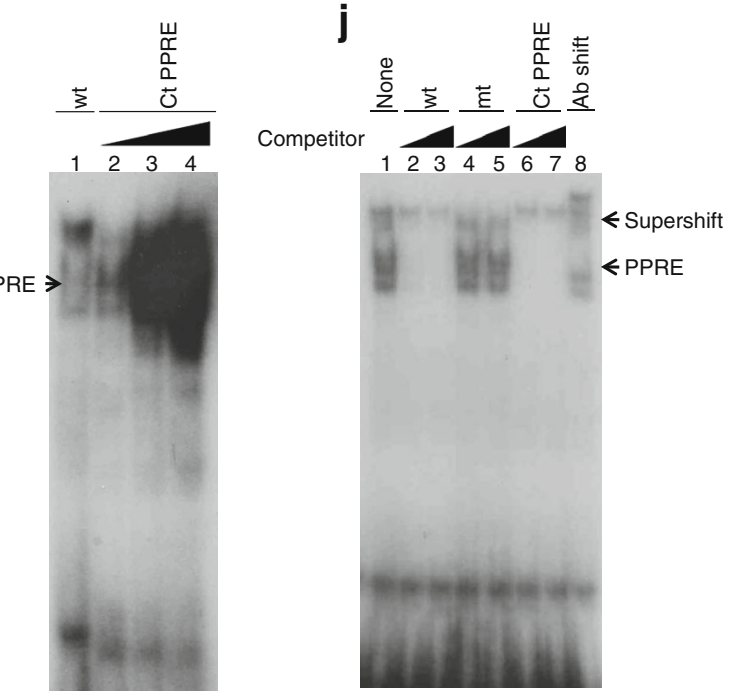

pGL2P-Bnip3-PPREwt or pGL2P-Bnip3-PPREmt, together with expression vectors of PPAR $\gamma$ and $\mathrm{pCMV}-\beta$-galactosidase, and normalised to $\beta$-galactosidase activity. Values of pGL2P-Bnip3-PPREwt or pGL2PBnip3-PPREmt without PPAR $\gamma$ and rosiglitazone were set at $1(n=3)$ (i, j) EMSA. (i) Binding assay for PPRE with a consensus PPRE oligomer as positive control with dose increase (lanes 2-4). (j) Unlabelled self (lanes 2,3) and mutant (lanes 4, 5) form of the $-2,874$ putative PPRE used for competition assay. Consensus PPRE oligomer was used as the positive control with dose increase (lanes 6,7). Antibody $(\mathrm{Ab})$ against PPAR $\gamma$ was added (lane 8). The PPRE site and supershift bands are marked with arrows. Data are presented as means \pm SE. All quantifications are compared with the reference value set at $1 .{ }^{*} p<0.05$

be critical in the deterioration of adipocyte function through mitochondrial dysregulation.

The role of BNIP3 during rosiglitazone-induced adipocyte remodelling was of significant interest in this study. We observed that the level of BNIP3 was lower under conditions associated with insulin resistance and was restored by rosiglitazone, a drug known to induce mitochondrial biogenesis $[2,7,10]$. We also found that BNIP3 expression increased primarily in newly developing multilocular adipocytes rather than dying adipocytes in rosiglitazone-treated $d b / d b$ mice (Fig. 2c) $[27,28]$. Therefore, BNIP3 might be involved in rosiglitazone-induced adipocyte remodelling rather than adipocyte death during the change in mitochondrial bioenergetics. The scale of BNIP upregulation in starvation 
was another interesting point. Even though we could not suggest the exact mechanism, we cautiously speculate that BNIP3 may play a role in maintaining the cellular ATP level and cell survival in the energy-deprived state caused by starvation [29]. Further investigation of this will be necessary.

\section{Adipocyte-specific role of BNIP3 in enhancement} of mitochondrial bioenergetics Originally, BNIP3 was reported to impair mitochondrial bioenergetics, as there were defects in mtDNA and ATP production in myocytes [30, 31]. However, our data show that BNIP3 enhances mitochondrial bioenergetics during rosiglitazone treatment, at least in adipocytes. Besides the increase in the amount of mitochondria, it was interesting to note that there was an increase in oxygen consumption and uncoupling, which suggests that BNIP3 enhances mitochondrial function in the adipocytes.

The critical differences in the effect of BNIP3 in various tissues might be because of differences in the tissue-specific expression and function of PPAR $\gamma$ [32-34], PPAR $\gamma$ coregulators [35, 36] and BNIP3-interacting proteins [37-39]. In fact, our preliminary experiments using $\mathrm{C} 2 \mathrm{C} 12$ myotubes showed the increment in mtDNA copy number resulting from rosiglitazone exposure to be independent of BNIP3 (data not shown). Also, different PPAR $\gamma$ co-regulators might enable BNIP3 to act in a tissue-specific manner $[35,36]$. Furthermore, from our bioinformatics analysis, we could find several mitochondria-related proteins that physically interact with BNIP3 [37-39] and are expressed differently depending on the type of tissue. These findings support our data showing a cell-context-dependent BNIP3 action and explain why the BNIP3-AMPK-PGC1 pathway acts differently in adipocytes in mitochondrial biogenesis.

We have also demonstrated that BNIP3 acts in a favourable way towards mitochondria activity rather than reducing it, at least in adipocytes. This adipocyte-specific action of BNIP3 is very interesting from an evolutionary viewpoint of energy regulation. Unlike BAT, which dissipates energy to produce heat, WAT stores energy. Recently, it has been shown that beige adipocytes sporadically reside with white adipocytes and emerge in response to certain environmental cues, such as chronic cold exposure, a process often referred to as 'browning' of WAT [40]. These findings suggest that beige fat in WAT and its energy dissipation function diminish during the evolution of thermogenic function. Therefore, our finding that BNIP3 plays an important role in enhancing mitochondria bioenergetics after PPAR $\gamma$ agonist stimulation could contribute to our understanding of the molecular mechanism of 'browning'.

Downstream pathway of BNIP3 in controlling mitochondrial remodelling Previously, BNIP3 has been shown to induce autophagy on exposure to rosiglitazone [41, 42]. However, we could not find any evidence of autophagy activation by
BNIP3 in adipocytes. Rather, rosiglitazone exposure decreased autophagy, even after BNIP3 upregulation (Fig. 4a, b). Therefore, BNIP3-mediated signalling in adipocytes might not act on autophagy regulation but may have a more intricate role during adipocyte remodelling. Here, it was interesting to find a possible role of $\mathrm{PGCl} \alpha$, a master transcription co-activator for mitochondrial biogenesis [43, 44], as a downstream molecule involved in PPAR $\gamma$-agonist-induced BNIP3 signalling in adipocytes. In our experiment, adiponectin was upregulated by rosiglitazone in a BNIP3-dependent manner. It is known that adiponectin can activate the AMPK-PGC $1 \alpha$ pathway, resulting in mitochondrial biogenesis [45]. Even though we could not definitively propose how BNIP3 mediates PGC1 $\alpha$ upregulation by rosiglitazone, we propose that adiponectinAMPK is one of the axes responsible. Considering that mitochondrial function is essential for adiponectin synthesis in adipocytes [46], BNIP3 might be a checkpoint for mitochondrial quality control [47] and the AMPK-PGC1 $\alpha$ mitochondrial bioenergetics pathway through which rosiglitazone acts. Further investigation of the precise mechanism of BNIP3-mediated mitochondrial biogenesis is warranted.

BNIP3 regulation by a PPAR $\gamma$ agonist in adipocytes So far, the regulation of BNIP3 expression has been focused on hypoxia inducible factor $1, \alpha$ subunit (basic helix-loop-helix transcription factor) (HIF-1 $\alpha$ ), a well-known transcription factor activated by hypoxia [16]. It is known that HIF-1 $\alpha$ upregulates BNIP3 and that the upregulated BNIP3 consequently activates autophagy, probably for survival against hypoxic stress [48]. Another molecule associated with BNIP3 regulation may be a PPAR $\gamma$ agonist, regardless of the increase in HIF- $1 \alpha$ or autophagy [49]. In our study, we found that Bnip 3 transcription is upregulated by a PPAR $\gamma$ agonist in adipocytes, irrespective of HIF-1 $\alpha$ (Fig. 5a, b). This is supported by the pattern of BNIP3 expression resembling that of PPAR $\gamma$ expression (Fig. 1c).

In conclusion, we propose a novel role for BNIP3 in mitochondrial bioenergetics in remodelling adipocytes and reveal a cell-context-dependent action of BNIP3 unique from that of other cells. The impairment in BNIP3 function in adipocytes might act to cause the mitochondrial bioenergetics to deteriorate and our finding suggests that a cell-type-specific measure to control BNIP3 may be a potential therapeutic target in obesity.

Acknowledgements We thank J.-W. Kim (Yonsei University College of Medicine, Seoul, Korea) for supplying the 3T3-L1 pre-adipocytes.

Funding This research was supported by the Korea Health Technology R\&D Project through the Korea Health Industry Development Institute (KHIDI), funded by the Ministry of Health \& Welfare, Republic of Korea (grant number: HI14C1277), and a faculty research grant from Yonsei University College of Medicine for 2011 (grant number: 6-2011-0152). 
Duality of interest The authors declare that there is no duality of interest associated with this manuscript.

Contribution statement SK, KSP, JWC and AJ contributed to the conception and design of the study. SK and KSP drafted the article. SSC, JWC, AJ, MK, HSP, SK and KSP contributed to the acquisition, analysis and interpretation of data, and revised the article critically for important intellectual content. All authors approved the final version. SK and KSP are the guarantors of this work and had full access to all the data in the study and take responsibility for the integrity of the data and the accuracy of the data analysis.

\section{References}

1. Sun K, Kusminski CM, Scherer PE (2011) Adipose tissue remodeling and obesity. J Clin Invest 121:2094-2101

2. Wilson-Fritch L, Nicoloro S, Chouinard M et al (2004) Mitochondrial remodeling in adipose tissue associated with obesity and treatment with rosiglitazone. J Clin Invest 114:1281-1289

3. Chan DC (2006) Mitochondria: dynamic organelles in disease, aging, and development. Cell 125:1241-1252

4. Duchen MR (2004) Mitochondria in health and disease: perspectives on a new mitochondrial biology. Mol Asp Med 25:365-451

5. Dahlman I, Forsgren M, Sjogren A et al (2006) Downregulation of electron transport chain genes in visceral adipose tissue in type 2 diabetes independent of obesity and possibly involving tumor necrosis factor-alpha. Diabetes 55:1792-1799

6. Heilbronn LK, Gan SK, Turner N, Campbell LV, Chisholm DJ (2007) Markers of mitochondrial biogenesis and metabolism are lower in overweight and obese insulin-resistant subjects. J Clin Endocrinol Metab 92:1467-1473

7. Rong JX, Qiu Y, Hansen MK et al (2007) Adipose mitochondrial biogenesis is suppressed in $\mathrm{db} / \mathrm{db}$ and high-fat diet-fed mice and improved by rosiglitazone. Diabetes 56:1751-1760

8. Chawla A, Schwarz EJ, Dimaculangan DD, Lazar MA (1994) Peroxisome proliferator-activated receptor (PPAR) $\gamma$ : adiposepredominant expression and induction early in adipocyte differentiation. Endocrinology 135:798-800

9. Spiegelman BM (1998) PPAR- $\gamma$ : adipogenic regulator and thiazolidinedione receptor. Diabetes 47:507-514

10. Wilson-Fritch L, Burkart A, Bell G et al (2003) Mitochondrial biogenesis and remodeling during adipogenesis and in response to the insulin sensitizer rosiglitazone. Mol Cell Biol 23:1085-1094

11. Bogacka I, Xie H, Bray GA, Smith SR (2005) Pioglitazone induces mitochondrial biogenesis in human subcutaneous adipose tissue in vivo. Diabetes 54:1392-1399

12. Feinstein DL, Spagnolo A, Akar C et al (2005) Receptorindependent actions of PPAR thiazolidinedione agonists: is mitochondrial function the key? Biochem Pharmacol 70:177-188

13. Vande Velde C, Cizeau J, Dubik D et al (2000) BNIP3 and genetic control of necrosis-like cell death through the mitochondrial permeability transition pore. Mol Cell Biol 20:5454-5468

14. Zhang J, Ney PA (2009) Role of BNIP3 and NIX in cell death, autophagy, and mitophagy. Cell Death Differ 16:939-946

15. Zhang HF, Bosch-Marce M, Shimoda LA et al (2008) Mitochondrial autophagy is an HIF-1-dependent adaptive metabolic response to hypoxia. J Biol Chem 283:10892-10903

16. Burton TR, Gibson SB (2009) The role of Bcl-2 family member BNIP3 in cell death and disease: NIPping at the heels of cell death. Cell Death Differ 16:515-523
17. Quan W, Lee MS (2013) Role of autophagy in the control of body metabolism. Endocrinol Metab (Seoul) 28:6-11

18. Baetz D, Regula KM, Ens K et al (2005) Nuclear factor-kappaBmediated cell survival involves transcriptional silencing of the mitochondrial death gene BNIP3 in ventricular myocytes. Circulation 112:3777-3785

19. Mammucari C, Milan G, Romanello Vet al (2007) FoxO3 controls autophagy in skeletal muscle in vivo. Cell Metab 6:458-471

20. Glick D, Zhang W, Beaton M et al (2012) BNip3 regulates mitochondrial function and lipid metabolism in the liver. Mol Cell Biol 32:2570-2584

21. Wu Y, Smas CM (2008) Wdnm1-like, a new adipokine with a role in MMP-2 activation. Am J Physiol Endocrinol Metab 295:E205E215

22. Bustin SA, Benes V, Garson JA et al (2009) The MIQE guidelines: minimum information for publication of quantitative real-time PCR experiments. Clin Chem 55:611-622

23. de Moura MB, Van Houten B (2014) Bioenergetic analysis of intact mammalian cells using the Seahorse XF24 extracellular flux analyzer and a luciferase ATP assay. Methods Mol Biol 1105:589-602

24. Keipert S, Jastroch M (2014) Brite/beige fat and UCP1 - is it thermogenesis? Biochim Biophys Acta 1837:1075-1082

25. Nisoli E, Tonello C, Cardile A et al (2005) Calorie restriction promotes mitochondrial biogenesis by inducing the expression of eNOS. Science 310:314-317

26. Houstek J, Kopecky J, Rychter Z, Soukup T (1988) Uncoupling protein in embryonic brown adipose tissue-existence of nonthermogenic and thermogenic mitochondria. Biochim Biophys Acta 935:19-25

27. Cinti S, Mitchell G, Barbatelli G et al (2005) Adipocyte death defines macrophage localization and function in adipose tissue of obese mice and humans. J Lipid Res 46:2347-2355

28. Koh YJ, Park BH, Park JH et al (2009) Activation of PPAR $\gamma$ induces profound multilocularization of adipocytes in adult mouse white adipose tissues. Exp Mol Med 41:880-895

29. Lopez-Lluch G, Hunt N, Jones B et al (2006) Calorie restriction induces mitochondrial biogenesis and bioenergetic efficiency. Proc Natl Acad Sci U S A 103:1768-1773

30. Kubli DA, Ycaza JE, Gustafsson AB (2007) Bnip3 mediates mitochondrial dysfunction and cell death through Bax and Bak. Biochem J 405:407-415

31. Grumati P, Bonaldo P (2012) Autophagy in skeletal muscle homeostasis and in muscular dystrophies. Cells 1:325-345

32. Adams M, Montague CT, Prins JB et al (1997) Activators of peroxisome proliferator-activated receptor gamma have depot-specific effects on human preadipocyte differentiation. J Clin Invest 100:3149-3153

33. Laplante M, Sell H, MacNaul KL, Richard D, Berger JP, Deshaies Y (2003) PPAR- $\gamma$ activation mediates adipose depot-specific effects on gene expression and lipoprotein lipase activity: mechanisms for modulation of postprandial lipemia and differential adipose accretion. Diabetes 52:291-299

34. Ahmadian M, Suh JM, Hah N et al (2013) PPAR $\gamma$ signaling and metabolism: the good, the bad and the future. Nat Med 19:557-566

35. Larsen TM, Toubro S, Astrup A (2003) PPAR $\gamma$ agonists in the treatment of type II diabetes: is increased fatness commensurate with long-term efficacy? Int J Obes Relat Metab Disord 27:147-161

36. Lessard SJ, Rivas DA, Chen ZP et al (2007) Tissue-specific effects of rosiglitazone and exercise in the treatment of lipid-induced insulin resistance. Diabetes 56:1856-1864

37. Landes T, Emorine LJ, Courilleau D, Rojo M, Belenguer P, Arnaune-Pelloquin L (2010) The BH3-only Bnip3 binds to the dynamin Opal to promote mitochondrial fragmentation and apoptosis by distinct mechanisms. EMBO Rep 11:459-465 
38. Li Y, Wang Y, Kim E et al (2007) Bnip3 mediates the hypoxiainduced inhibition on mammalian target of rapamycin by interacting with Rheb. J Biol Chem 282:35803-35813

39. Burton TR, Eisenstat DD, Gibson SB (2009) BNIP3 (Bcl-2 19 kDa interacting protein) acts as transcriptional repressor of apoptosisinducing factor expression preventing cell death in human malignant gliomas. J Neurosci 29:4189-4199

40. Sidossis L, Kajimura S (2015) Brown and beige fat in humans: thermogenic adipocytes that control energy and glucose homeostasis. J Clin Invest 125:478-486

41. Cerquetti L, Sampaoli C, Amendola D et al (2011) Rosiglitazone induces autophagy in H295R and cell cycle deregulation in SW13 adrenocortical cancer cells. Exp Cell Res 317:1397-1410

42. Wu J, Wu JJ, Yang LJ, Wei LX, Zou DJ (2013) Rosiglitazone protects against palmitate-induced pancreatic beta-cell death by activation of autophagy via 5'-AMP-activated protein kinase modulation. Endocrine 44:87-98

43. Finck BN, Kelly DP (2006) PGC-1 coactivators: inducible regulators of energy metabolism in health and disease. J Clin Invest 116:615-622

44. Lehman JJ, Barger PM, Kovacs A, Saffitz JE, Medeiros DM, Kelly DP (2000) Peroxisome proliferator-activated receptor gamma coactivator-1 promotes cardiac mitochondrial biogenesis. J Clin Invest 106:847-856

45. Shen Z, Liang X, Rogers CQ, Rideout D, You M (2010) Involvement of adiponectin-SIRT1-AMPK signaling in the protective action of rosiglitazone against alcoholic fatty liver in mice. Am J Physiol Gastrointest Liver Physiol 298: G364-G374

46. Koh EH, Park JY, Park HS et al (2007) Essential role of mitochondrial function in adiponectin synthesis in adipocytes. Diabetes 56:2973-2981

47. Palikaras K, Tavernarakis N (2014) Mitochondrial homeostasis: the interplay between mitophagy and mitochondrial biogenesis. Exp Gerontol 56:182-188

48. Bellot G, Garcia-Medina R, Gounon P et al (2009) Hypoxiainduced autophagy is mediated through hypoxia-inducible factor induction of BNIP3 and BNIP3L via their $\mathrm{BH} 3$ domains. Mol Cell Biol 29:2570-2581

49. Zhou J, Zhang W, Liang B et al (2009) PPAR $\gamma$ activation induces autophagy in breast cancer cells. Int $\mathrm{J}$ Biochem Cell Biol 41:2334-2342 